Supplement of Solid Earth, 7, 1383-1393, 2016

http://www.solid-earth.net/7/1383/2016/

doi:10.5194/se-7-1383-2016-supplement

(C) Author(s) 2016. CC Attribution 3.0 License.

(c) (i)

Supplement of

\title{
Eruptive shearing of tube pumice: pure and simple
}

Donald B. Dingwell et al.

Correspondence to: Yan Lavallée (ylava@liverpool.ac.uk)

The copyright of individual parts of the supplement might differ from the CC-BY 3.0 licence. 


\section{Neutron tomography image analysis}

To extract a bubble for further analysis, the resulting dataset was then 3 segmented using a watershed algorithm acting on the attenuation gradient (Banhart, 4 2008). This method begins by allocating a unique label to all local minima in the 5 gradient. The remaining voxels are inserted into a priority queue. The priority of each 6 voxel is inversely proportional to its gradient, i.e. low gradient voxels are processed 7 first. Each un-labelled voxel in the queue is tested to see if any of its neighbours are 8 labelled. If any of the neighbours of this voxel have already been labelled, and all of 9 those labels are the same, then the voxel is allocated this label. If two or more of the 10 neighbourhood labels are different then this voxel will be labelled as an "edge". This 11 edge definition is very rigorous as it always defines the edge at the position of 12 maximum gradient between two objects. The edge also forms a perfectly closed shell 13 around each object and this can be viewed by discriminating individual bubbles (Fig. $147)$.

15 Watershed segmentation is sensitive to image noise, as small perturbations in 16 the image intensity can generate local minima and lead to over-segmentation. This 17 was counteracted using de-speckling, or "median" filtering in a $3 \times 3 \times 3$ voxel 18 neighbourhood to remove high amplitude noise (e.g., Banhart, 2008). Low-amplitude 19 noise in the attenuation gradient was removed using an H-minima transformation 20 (e.g., Banhart, 2008). This suppresses all minima whose depth is less than a specified 21 cut-off level.

\section{References}

24 Banhart, J.: Advanced tomographic methods in materials research and engineering, 25 Oxford University Press, New York, 2008. 